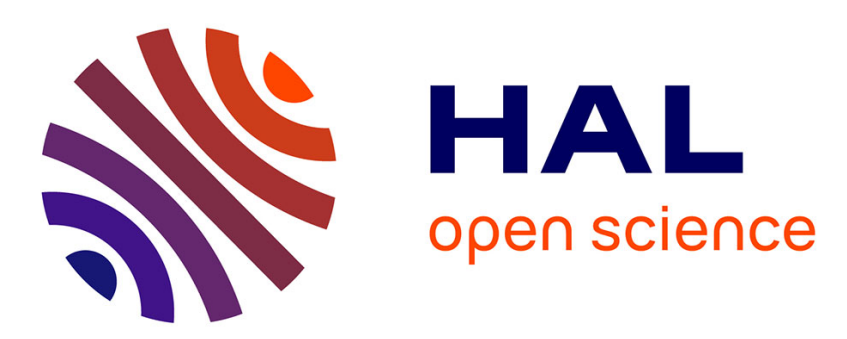

\title{
Formes et enjeux de la rétrospection
}

Christian Puech, Valérie Raby

\section{To cite this version:}

Christian Puech, Valérie Raby. Formes et enjeux de la rétrospection: Présentation. Histoire Epistémologie Langage, 2011, " Histoire de la linguistique et horizons de rétrospection II ", 33 (2), pp.5-14. 10.3406/hel.2011.3217 . hal-01378227

\section{HAL Id: hal-01378227 https://hal.science/hal-01378227}

Submitted on 9 Oct 2016

HAL is a multi-disciplinary open access archive for the deposit and dissemination of scientific research documents, whether they are published or not. The documents may come from teaching and research institutions in France or abroad, or from public or private research centers.
L'archive ouverte pluridisciplinaire HAL, est destinée au dépôt et à la diffusion de documents scientifiques de niveau recherche, publiés ou non, émanant des établissements d'enseignement et de recherche français ou étrangers, des laboratoires publics ou privés. 


\section{Présentation. Formes et enjeux de la rétrospection}

Christian Puech, Valérie Raby

\section{Citer ce document / Cite this document :}

Puech Christian, Raby Valérie. Présentation. Formes et enjeux de la rétrospection. In: Histoire Épistémologie Langage, tome 33, fascicule 2, 2011. Histoire des idées linguistiques et horizons de rétrospection - II. pp. 5-14;

doi : 10.3406/hel.2011.3217

http://www.persee.fr/doc/hel_0750-8069_2011_num_33_2_3217

Document généré le 15/06/2016 


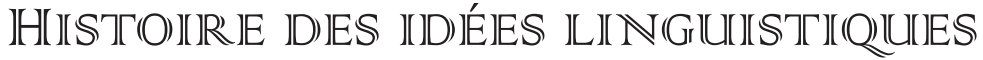

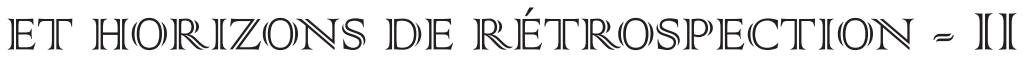

\section{FORMES ET ENJEUX DE LA RÉTROSPECTION PRÉSENTATION}

\section{Christian Puech \& Valérie Raby}

UMR 7597 HTL Université Sorbonne Nouvelle - PRES Sorbonne Paris Cité \& UMR 7597 HTL Université Paris-Sorbonne (Paris IV)

\author{
Nous dédions ce dossier auquel elle devait contribuer \\ à notre regrettée collègue et chère amie \\ Sophie Kessler-Mesguich \\ (6 décembre1957- 8 février 2010).
}

Ce deuxième dossier consacré aux «horizons de rétrospection » des théories linguistiques poursuit l'entreprise entamée dans le tome XXVIII, fascicule 1 de Histoire Épistémologie Langage (2006). Plus exactement, il cherche à compléter et approfondir la recherche historiographique concernant « l'histoire de la linguistique dans l'histoire de la linguistique » tout en en infléchissant de manière sensible le parcours.

Très schématiquement, le précédent numéro visait, d'une part, à situer la recherche historiographique autour de quelques thématiques liées à l'histoire des sciences et, à l'intérieur du domaine, à un bilan au moins sommaire des acquis de l'histoire de la linguistique. Pourquoi dans ce cadre s'intéresser à l'histoire des représentations de l'histoire des sciences du langage au sein de l'histoire des idées linguistiques? Qu'est-on en droit d'en attendre ? À quoi doit-on renoncer ? Au- 
delà de la recherche érudite (indispensable), quelles en sont les limites ? Quels sont les cadres généraux susceptibles d'aider à comprendre les continuités et renouvellements des théorisations? Si l'on admet que la temporalité est l'une des dimensions fondamentales des théories du langage, quel rôle joue-t-elle dans la conscience des différents acteurs de cette histoire ? Dans quelle mesure cette conscience partielle/partiale relève-t-elle elle-même de plein droit de l'histoire des idées linguistiques ? (cf. C. Puech 2006).

Le dossier précédent cherchait d'autre part à « sonder » différentes périodes de cette histoire en essayant de faire ressortir à la fois les constantes et les changements dans la manière dont les grammairiens/linguistes ont recours à l'idée (simple) selon laquelle les idées/descriptions/explications qu'ils développent concernant les langues sont elles-mêmes affectées par une temporalité qui les détermine pour une part et qu'elles reflètent réflexivement de manières différentes selon les époques, les lieux, les cadres généraux variables, les intérêts de connaissance investis.

Sans pouvoir développer suffisamment ni de manière égale, nous croyons avoir fait apparaître ou avoir confirmé, de la Renaissance à la fin du $19^{\mathrm{e}} \mathrm{s}$., un certain nombre de constantes et un certain nombre de changements.

\section{HISTOIRE ET PROGRÈS}

Le premier point est le rôle de seuil que semble jouer le $18^{\mathrm{e}}$ siècle dans l'émergence d'une conscience radicale et moderne de la temporalité (en général, bien sûr, mais aussi dans les sciences du langage) : ici, le temps devient cause dans une chaîne à la fois déterminante et irréversible. Si l'on suit Auroux/Colombat (1999) à propos des citations de l'Encyclopédie, la fonction «d'oubli » devient alors et complémentairement décisive : les auteurs notent la quasi-absence de références au Moyen Âge, la mention étonnamment discrète des grammairiens humanistes de la Renaissance et la survalorisation corrélative de l'Antiquité. On peut voir là, d'une part, un horizon de rétrospection (qui se confond avec une stratégie mémorielle) ; on peut voir également, d'autre part, le lien existant entre le point de vue historique et le point de vue encyclopédique : la nécessité ressentie alors de construire une saisie globale qui intègre les différents moments du développement de la discipline en une représentation disciplinaire qui soit à la fois une synthèse des « acquis » et une vision prospective (cf. Fournier/Raby 2006) ${ }^{1}$. Cette double caractéristique implique nécessairement que les idées linguistiques sont, à partir d'un certain seuil, et avec d'autres domaines de connaissance (mathématiques, médecine, astronomie, chimie, physique...), affectées par la notion de progrès ${ }^{2}$.

1 «Le testament, dans la mesure où il dit à l'héritier ce qui sera légitimement sien, assigne un passé à l'avenir » (Arendt 1972).

2 On a souvent dit que l'histoire des sciences moderne commençait avec B. de Fontenelle (1740) et ses Éloges des Académiciens avec l'histoire de l'Académie royale des sciences en 1699, où l'on lit par exemple : "Il est très agréable, et ce plaisir renferme beaucoup d'instruction, de voir la route que l'esprit humain a tenue et, pour parler géométriquement, cette espèce de progression, dont les intervalles sont d'abord grands, et vont ensuite naturellement en se serrant toujours plus » (t. 1, p. 148, nous soulignons). Conformément à son étymologie, l'histoire fait « voir ». A. Zangara (2007) développe pour les théories anciennes du récit historique tous les paradoxes de la vision «par procuration », de « l'autopsia » historique. Et d'abord celui-ci : quelle sorte de présent est ce «présent » que rend accessible l'histoire 
De ce point de vue, l'apparition d'un discours «spécial» qui se met en place dans la deuxième moitié du $19^{\mathrm{e}} \mathrm{s}$. dans des préfaces ou des ouvrages spécialisés, consacre à notre sens une étape pour laquelle références scientifiques «actives » et « références historiques » viennent alors à se distinguer, sans bien sûr s'exclure non plus totalement.

On peut légitimement penser que c'est cette notion de progrès, avec sa teneur en continuité et discontinuité, en synthèses et disjonctions qui informe, engage et perturbe à la fois les représentations de l'histoire de la linguistique depuis les années 1960 au moins. C'est sur ce point que nous souhaitons revenir maintenant, dans la mesure où nous pensons que la spécificité de ce qu'on appelle parfois la « tradition française de l'épistémologie historique », en tant, précisément, qu'elle est bien «historique », et donc qu'elle entretient avec l'histoire des rapports particulièrement forts - mais peut-être aussi paradoxaux - est concernée au premier chef par la question de «l'histoire dans l'histoire» (cf. E. Castelli Gattinara, 1998).

Dans ce cadre, et conformément à la double postulation dont est affectée cette enquête sur l'histoire de la linguistique dans l'histoire de la linguistique, l'enjeu est double : (A) d'une part, il concerne ce que S. Auroux (2006) appelle le processus d'historicisation des connaissances linguistiques. La temporalité du développement des idées linguistiques intervient en effet plusieurs fois :

i) une fois au niveau de l'objet même selon que les descriptions / explications des faits linguistiques tiennent ou ne tiennent pas (ou peu) compte selon telle ou telle modalité (impliquant l'histoire des langues ou ne l'impliquant pas ou peu) de la constitution des faits linguistiques dans le temps. Or, et pour prendre un exemple qui nous est contemporain, le concept de " grammaticalisation », aujourd'hui particulièrement souligné dans les recherches, et le rôle assigné à ce concept dans l'histoire des langues et dans leur fonctionnement « actuel » (sa dimension cognitive), les débats qu'il peut susciter au regard d'une conception générale du type de temporalité qui régit l'histoire des langues (sa teneur en « téléologie »), mais aussi des révisions qu'il entraine concernant ce qu'on doit entendre par « diachronie » (cf. M.-J. Béguelin 2010), montrent bien que les représentations du temps linguistique affectent d'un même mouvement, et la conception de l'objet, et la configuration de la discipline qui prétend le prendre en charge.

ii) La temporalité intervient également au niveau des effets en retour linguistiques de la grammatisation: la «réduction de la langue en règles » affecte les pratiques langagières elles-mêmes selon des modalités (sociales, historiques et symboliques) repérables, même si elles varient dans le temps et l'espace. Elle rend donc également accessibles, parfois, des données de langue disparues auxquelles ces modalités ont donné forme et consistance dans des traditions continues qui constituent comme des «fabriques » de langue.

concernant des faits passés ? Si l'on suit ce fil, comment intégrer l'idée de progrès (ces intervalles qui ne cessent de raccourcir en se rapprochant de nous) dans celle d'histoire ? La question du présentisme est sans doute au cœur de toute histoire des sciences dans l'acception moderne de ce terme. Elle nous semble parcourir l'ensemble des contributions à ce dossier. 
(B) D'autre part et en conséquence enfin, le processus de construction de représentations secondes de ces «processus primaires » dans des récits, des pratiques de restitution ouverts à la fois à la rétention (dilatation du présent vers le passé) et la protention (dilatation du présent vers le futur), sujets également à révisions et corrections, investissements, réinvestissements et désinvestissements eux aussi variables dans l'espace et dans le temps, ouvre à la possibilité de «mises en intrigue », de sortes de «scénarisations », donc, de l'histoire des savoirs. C'est ce processus de reconstruction, spontané ou érudit, plus ou moins engagé ou non dans la construction actuelle de ces savoirs, indexé souvent de manière étroite sur l'histoire «externe » (cf. par ex., pour la tradition russe, S. Archaimbault 2006), qui préside aux diagnostics de «crise » (D. Savatovsky 2006 pour la fin du $19^{\mathrm{e}} \mathrm{s}$.), dessine pour les acteurs l'actualité et l' avenir de leur pratique de connaissance, guide et oriente leur évaluation du champ, tout en en délimitant à leurs yeux les contours légitimes. Il va bien sûr de soi que c'est dans cette construction de représentations de l'histoire - où l'on peut penser que tous les scénarios de reconstructions ne s'équivalent pas - que se jouent pour une part le sens de l'activité historienne ainsi que l'impact éventuel de l'histoire de la linguistique sur les développements actuels de la discipline.

Comme nous le notions, la question reste entière de savoir comment et/ou dans quelle mesure cette implication du temps dans les théorisations du langage relève de l'historiographie au sens le plus traditionnel du terme ou d'une «métahistoriographie » que plusieurs spécialistes du domaine tentent de promouvoir depuis plusieurs années (cf. en particulier P. Schmitter et M. van der Wal eds 1998, C. Puech 2006).

\section{LES FILIATIONS DOXOGRAPHIQUES}

Quoi qu'il en soit, il est possible et utile de poursuivre l'enquête en l'étendant d'abord à d'autres traditions.

Jean-Patrick Guillaume pour la tradition arabe, Jean-Luc Chevillard pour la tradition tamoule poursuivent l'investigation en nous confrontant à une familière étrangeté : les doxographies des grammairiens arabes et tamouls ne se ressemblent évidemment pas. Elles ne ressemblent pas non plus aux doxographies grécolatines de la Renaissance dont nous entretenait B. Colombat (2006), ni à celles dont fait état Anne Grondeux pour le Moyen Âge dans ce numéro. Il faut donc à chacun de nos auteurs une expertise spéciale pour reconstruire ces systèmes de référence au passé. Chacun de ces systèmes s'appuie en effet sur une technique qui lui est propre mais qui peut être aussi diffuse, sur une terminologie la plupart du temps non stabilisée (veteres, antiqui, moderni au Moyen Âge, par exemple). Les disputes et controverses auxquelles ils renvoient sont parfois « anonymisées », sans indexation chronologique, sans référence explicite à des écoles (dans la tradition arabe par exemple, mais aussi chez les médiévaux). Ils peuvent également tout simplement ne se référer au passé que pour conjurer le changement et garantir la permanence (la «perpétuité ») de leur objet au prix d'artifices techniques qu'ils masquent consciencieusement pour escamoter les contradictions trop visibles, comme le montre Jean-Luc Chevillard en ce qui concerne le savoir métrique et 
grammatical tamoul transmis sur une période de mille cinq cents ans.

Et pourtant, ces modes de référence au passé sont-il si éloignés les uns des autres ? L'effort des contributeurs de ce dossier pour les décrire (et nous les expliquer) dans leur logique, in situ, montre une fois de plus que l'incommensurabilité des systèmes explicatifs n'est que relative : l'horizon des textes sacrés, instance ultime de légitimation, cible de toute explication et fondement de toute Autorité, leur confère sans doute un «air de famille ». Mais ce n'est peut-être pas le seul point de contact. Il nous paraît frappant, par exemple, que le fondement de l'autorité ne semble se trouver pour aucune de ces traditions dans la seule ancienneté de la référence (Donat ou Priscien, le Tolkäppiyam tamoul...). C'est que les dispositifs de légitimation doivent bien ménager la place à de nouvelles autorités qui ne seront établies toutefois qu'à la condition qu'elles exhibent la preuve de leur affiliation (A. Grondeux, J.-P. Guillaume, J.-L. Chevillard). Au-delà des différences évidentes entre le recours médiéval au passé et celui des traditions arabes et tamoules, l'horizon de rétrospection prend donc déjà l'aspect d'une négociation entre la permanence et le changement. Ce dernier n'est pas fondamentalement nié, mais il est intégré à une représentation du temps où la valeur du « moderne » ne saurait lui être conférée que par la tradition vis-à-vis de laquelle le «moderne » contracte ainsi une dette... et donc, dans une circularité de valorisations réciproques sans terme.

Au fond, Jean-Luc Chevillard ne nous livre-t-il pas l'exemple d'une organisation sophistiquée de la mémoire dont le but est avant tout de conjurer le changement dans un univers intellectuel soumis aux contraintes fortes de la ritualisation? Le rôle de l'historien étant ici de rétablir - non sans mal - des formes d'intelligibilité liées au contraire à l'irréversibilité du temps et à la diversification inéluctable des objets d'étude.

\section{LES STRATÉGIES MÉMORIELLES}

L'enquête s'ouvre ici également, nous semble-t-il, à une possible typologie des « usages du passé »- pour reprendre une dénomination consacrée de longue date chez les historiens - en faisant varier encore et l'empan historique considéré (Moyen Âge, A. Grondeux) et la diversité des contextes dans lesquels des grammairiens (H. Merlin-Kajman), des linguistes (C. Blanckaert) ou des philologues (P. RabaultFeuerhahn) pensent leur propre activité à différentes périodes.

De ce point de vue, et modulo, là encore, des différences qui rendent l'exercice de comparaison bien périlleux, on pourrait isoler deux cas extrêmes :

i) En se donnant pour objet lareprésentation du paradigmenaturaliste (organiciste) tel qu'il est construit par l'historiographie des sciences du langage au tournant des $19^{\mathrm{e}}$ et $20^{\mathrm{e}}$ siècles (M. Bréal et F. de Saussure en particulier) et relayé par les discours de vulgarisation sur l'histoire de la linguistique, Claude Blanckaert choisit l' examen d'une histoire à moyen terme, fortement engagée dans une lutte pour la prééminence, et d'allure agonistique. D'un côté en effet le paradigme linguistique concurrent - «historico-social » - tenterait de promouvoir une histoire à visée d'auto-légitimation par censure, exclusion et recentrage dont le corrélat, de l'autre, procèderait par capitalisation et canonisation des sources. Dans la postérité de Bréal et Saussure, la condamnation des naturalismes se 
serait adjoint la sanction morale, faisant valoir la faute qui aura consisté à installer et légitimer, comme avant terme, une forme de racialisme linguistique. Selon C. Blanckaert, le présentisme historique se manifeste ici par le caractère essentiellement épidictique du discours historique. Par là, l'historiographie rejoint la mémoire vive disciplinaire toujours menacée de dégénérer en une science des manuels qui est en quelque sorte une histoire des «vainqueurs » sans profondeur de champ et outrageusement homogénéisatrice. C. Blanckaert alerte en somme l'historien contre nos déficits dans l'approche des théories condamnées ou controversées. Concernant la linguistique naturaliste, c'est notre défaut d'appréciation de la culture commune, des références partagées, du « dispositif cognitif global » au $19^{e}$ s. entre la géologie, la philologie, les sciences de la société et les sciences de la vie qui est en cause. Contre cet usage étroitement disciplinaire auto-légitimateur du passé, ne retrouve-t-on pas l'injonction encyclopédique de reconstruction d'un dispositif cognitif global ? Mais si l'on procède ainsi, comment échapper au fameux «principe de symétrie » de l'Ecole d'Edimbourg, pour lequel le fondement social des connaissances scientifiques explique également la production du faux et celle du vrai ?

ii) En tentant de suivre les linéaments sinueux de l'interprétation donnée au cours des temps de l'article 111 de l'Édit de Villers-Cotterêts, l'histoire pratiquée par H. Merlin-Kajman ne se situe pas seulement dans le long terme d'une histoire trop vite jugée qui demanderait rectification par souci de justice. Elle s'installe dans un espace polycentrique de représentations où les considérations juridiques, sociales, les conceptions de la souveraineté politique le disputent - en s'articulant à elles - aux enjeux linguistiques... et historiques. Elle touche donc, sur un point particulièrement sensible - la reconnaissance de ce que l'on peut entendre par « langage maternel français »-, à la dimension symbolique et fortement performative du travail du grammairien linguiste, et par extension, à celui de l'historien des idées linguistiques. Elle engage non seulement une conception de l'histoire des idées linguistiques, mais aussi celle d'une histoire « globale » de l'institution du français entendue non comme un acte ponctuel, mais comme un processus qui mobilise plusieurs siècles dans la « fabrique de la langue » (Auroux et Mazière 2008) et prolonge ses effets jusqu'à notre présent.

En effet, l'ordonnance de Villers-Cotterêts n'inaugure rien de nouveau, selon H. Merlin-Kajman. Elle met « simplement » de l'ordre en périmant une marque de la domination romaine, à une époque où « l'historicisation du temps commence à faire de l'empire romain un moment historique révolu ». Dans cette mesure, si « la restitution du français dans les arrêts est le signe d'une dignité recouvrée », comment rendre compte de cette perpétuité largement symbolique qui met en contraste une langue perpétuelle, mais sans locuteur (le latin), et une langue « métisse » et liée à la contingence d'un roi et d'un peuple, mais animée d'un désir de perpétuité (le français) ? «Il suffit - répond H. Merlin-Kajman - de locuteurs vivants et conscients de la dimension symbolique d'un tel partage pour que l'impossible appel de Valla se transforme en tâche historique possible », et pour ouvrir donc, un cycle d'avenir ouvert aux réinterprétations prometteuses 
des origines comme aux abîmes de l'oubli et de l'oubli de l'oubli...

On dira peut-être que l'enjeu dépasse ici largement ceux de l'histoire des sciences du langage, mais on peut penser aussi que l'histoire des sciences du langage, parce qu'elle touche à un objet qui concerne de manière prototypique l'ensemble des productions symboliques, doit nécessairement s'affronter à cette dimension qui lui revient de plein droit, comme lui revient l'étude des processus aux enjeux éminemment symboliques de nomination des langues (cf. Aussant 2009 et Tabouret Keller 1997) dont l'enquête d'H. Merlin-Kajman se rapproche asymptotiquement.

\section{L'HISTOIRE COMME « RECUEIL »}

Toutes les contributions à notre dossier confirment l'importance de l'histoire comme rassemblement, cette fonction de rassemblement étant ressentie comme particulièrement nécessaire à certains moments. Il s'agit alors de lutter contre la dispersion des sources, d'établir l'index des « hauts faits » de la discipline, de marquer des frontières et des lignes de partages, de s'assurer de la consistance de l'objet dont on traite. C'est là la fonction des notions d'《héritage » et d'«autorité » dans les doxographies antiques, médiévales, renaissantes, arabes, tamoules (dans d'autres sûrement aussi) : le cumul de références qui n'ont pas elles-mêmes de références (des origines sans origines) inclut et conjure à la fois les risques du changement et de l'innovation. Il garantit des formes de perpétuité dont l'appréhension du statut « historique » ne peut faire l'économie de la dimension symbolique. Il ne s'agit pas seulement alors de remonter aux sources, mais d'instituer un ordre de légitimité.

On aurait tort de penser que cette configuration ne concerne que des temps anciens ou des lieux exotiques. Dès la Renaissance, l'efficacité du symbolique affecte la représentation historique quand, par exemple, et au terme des chapitres qui insèrent les textes du Serment de Strasbourg, Claude Fauchet (1581) évoque cette «langue que j'appelle française », et en déduit l'existence passée de cette langue, sciemment altérée par les conquérants romains (H. Merlin-Kajman). Ce faisant, cet acte de nomination en partie performatif superpose de manière indissociable antériorité historique et primauté légitime.

A fortiori, ce qui concerne l'objet étudié concerne aussi, à l'époque contemporaine, à l'âge de la science, les disciplines qui l'étudient. L'exemple traité par C. Blanckaert le montre de manière saillante : la contrainte du « progrès » présuppose presque mécaniquement l'auto-promotion de la nouveauté. Or, celle-ci ne saurait s'instituer sans un combat victorieux et le rassemblement d'un camp contre un autre dont on homogénéise l'existence à tout prix. Dans le combat contre les naturalistes à la charnière des $19^{\mathrm{e}}$ et $20^{\mathrm{e}}$ siècles, on pourrait exhiber mille reprises ou paraphrases de formules qui n'ont plus besoin d'être rapportées à un premier énonciateur reconnu : la formule « le langage n'est pas un quatrième règne de la nature » circule entre Bréal, Saussure, Paris, Meillet, Mounin (et sans doute beaucoup d'autres) comme une évidence anonyme. La référence, par-delà «l'horizon d'exécration » stigmatisé, à «nos pères de l'Ecole de Condillac » circule là encore en effet de Bréal à Saussure et à Meillet, à la fois comme un 
contrepoint et un paradis perdu, d'autant plus dévalorisant pour l'école naturaliste qu'il ne lui est jamais opposé sans être accompagné d'un même mouvement de restriction et concession (« nos pères de l'école de Condillac avaient au moins le mérite... »).

Pascale Rabault-Feuerhahn, en étudiant l'historiographie de l'indianisme, explore enfin encore une autre configuration de la mémoire disciplinaire. À la charnière des $18^{\mathrm{e}}$ et $19^{\mathrm{e}}$ siècles, la philologie indienne se constitue en écrivant, comme «en marchant», sa propre histoire et double le discours de la nouveauté d'un discours de légitimité historique. La rétrospection s'inscrit ici en effet dans un triple contexte avec un triple but :

i) accompagner l'émergence de la nouveauté d'un discours d'escorte susceptible de convaincre un large public (la mode indienne) ainsi que des commanditaires détenteurs de financements (l'organisation novatrice de l'université allemande),

ii) installer l'Allemagne dans un statut de leadership gagné sur le colonisateur anglais plus proche du «terrain », certes, mais justement trop intéressé et pragmatique pour développer pleinement un savoir désintéressé, gratuit, conforme aux normes d'une « science occidentale » pensée à la fois comme le complément et comme l'autre des savoirs de l'Inde.

iii) affirmer l'affinité profonde et naturelle de l'Allemagne et de l'Inde.

Cette histoire de combat parée des vertus de la science se développe d'abord à partir des acteurs eux-mêmes se tendant à eux-mêmes un miroir : préfaces, notules, biographies de héros scientifiques, jubilées universitaires, notices nécrologiques, jettent les bases d'une scénographie à la recherche de sa dramaturgie. Dans ce cadre, linguistique (Humboldt, Bopp...) et philologie interprétative, spéculative (Schlegel...) s'épaulent pour s'assurer une supériorité sans partage (mais non sans collaborations) sur la science de terrain (la science anglaise).

Les monographies historiographiques viennent donc (beaucoup) plus tard, mais le décor est d'emblée planté, les caractères des héros sont définis et le registre de l'action émerge : ce sera celui de l'épopée, du récit de voyage ou d'exploration. La bibliothèque, cet équivalent du terrain exotique, devient le locus terribilis qui fournit le cadre d'une geste épique accomplie d'abord par des héros-chercheurs isolés qui fournissent la généalogie fondatrice d'un domaine censé être la réplique virtuelle d'un empire réel (l'empire anglais), puis permettant de rendre visible une communauté d'autant plus facilement identifiable qu'elle se confond avec l'Occident même...

En dernier ressort - écrit Pascale Rabault-Feuerhahn - la mise en scène historiographique de l'indianisme comme épopée ne participe pas simplement d'une représentation naïvement positiviste et cumulative de l'histoire des sciences, mais aussi et surtout d'une confiance inébranlable dans les pouvoirs de la philologie occidentale - confiance encore confortée par le recours au comparatisme, censé à la fois incarner la modernité scientifique et confirmer la légitimité particulière des Allemands à se prononcer sur l'histoire culturelle indienne. 


\section{DEMAIN ?}

On pourrait certainement accrôtre encore l'empan historique et la diversité des « coups de sonde » lancés à la recherche des « formes et enjeux de la rétrospection ». Mais notre but n'était pas ici de prétendre à une quelconque exhaustivité, plutôt de contribuer à situer la pratique historienne dans sa propre histoire. Conclure serait hasardeux et n'est donc pas nécessaire. On peut toutefois envisager les prolongements de ce chantier qui montre à notre sens plusieurs choses :

i) La première est sans doute que ce retour critique de la pratique historienne sur elle-même n'est, malgré sa sophistication apparente, ni un luxe ni un moment d'exception dans le travail quotidien de l'historien. Les théories linguistiques à la généalogie desquelles il se consacre n'ont pu lui parvenir qu'à travers une multiplicité de canaux qui sont autant de filtres contribuant à leur donner leur physionomie à ses yeux. Comme entreprise critique et réflexive, l'histoire des idées linguistiques doit selon nous sans cesse revenir sur la diversité des formes de temporalité qui met à sa disposition ses objets d'études et ses protocoles de recherche et de vérification.

ii) L'idée d' «horizon de rétrospection » procède au fond d'une idée simple : l'historien ne peut penser que les connaissances (les connaissances linguistiques comme les autres) seraient produites dans un temps homogène au rythme régulier et selon une progression géométrique telle que l'évoque Fontenelle. Mais si l'idée de connaissance ponctuelle (le vieux préjugé d'évidence et d'intuition) doit être abandonnée, si l'image de la connaissance comme succession de points entretenant entre eux une distance relative doit être tempérée également sans être radicalement exclue, l'exploration du présent de la connaissance demande lui à être approfondi dans son ambivalence même. Ce présent est nécessairement un présent de la co-présence qui met en relation plusieurs moment du temps et fait que toute référence à un passé n'est pas encore de l'histoire.

iii) Enfin, la place nous a manqué pour le faire, mais le lecteur se rendra compte par lui-même que bien des contributions à ces deux dossiers d'Histoire Épistémologie Langage font nécessairement allusion, en relation étroite avec les horizons de rétrospection, aux horizons de projection de la discipline. Cet aspect de l'histoire des idées linguistiques ne mériterait-il pas à lui seul une étude spéciale ? Si l'histoire «fait voir » c'est en plusieurs sens de "voir», dont l'un semble comme explicité par la fameuse formule de Michelet selon laquelle «chaque époque rêve la suivante ». Quels ont été les formes et enjeux des avenirs projetés des sciences du langage ? Quels sont-ils aujourd'hui ? 


\section{BIBLIOGRAPHIE}

Archaimbault, Sylvie (2006). «L'histoire de la linguistique, un élément d'une culture linguistique nationale », Histoire Épistémologie Langage 28/1, 77-88.

Arendt, Hannah (1961 [1972]). Between Past and Future : Six Exercices in Political Thought, New York, The Viking Press - trad. La Crise de la culture, Paris, Gallimard.

Auroux, Sylvain (2006). «Les modes d'historicisation », Histoire Épistémologie Langage 28/1, 105-116.

Auroux, Sylvain et Colombat, Bernard (1999). «L'horizon de rétrospection des grammairiens de l'Encyclopédie », Recherches sur Diderot et l'Encyclopédie 27 (octobre 1999), 111-152.

Auroux, Sylvain et Mazière, Francine (dir.) (2008). «Hyperlangues et fabriques de langues », Histoire Épistémologie Langage 28/2.

Aussant, Émilie (dir.) (2009). «La nomination des langues dans l'histoire », Histoire Épistémologie Langage 31/2.

Béguelin, Marie-José (2010). «Le statut des identités diachroniques dans la théorie saussurienne. Une critique anticipée du concept de grammaticalisation », J.-P. Bronckart, C. Bota \& E. Bulea (éds), Le projet de Ferdinand de Saussure, Genève, Droz, 237-267.

Castelli Gattinara, Emilio (1998). Les inquiétudes de la raison : épistémologie et histoire en France dans l'entre-deux guerres, Paris, Vrin / EHESS.

Colombat, Bernard (2006). "Les références aux anciens et aux modernes chez les grammairiens latins du $16^{\mathrm{e}}$ siècle (Linacre, Scaliger, Ramus, Sanctius) », Histoire Épistémologie Langage 28/1, 25-50.

Fontenelle, Bernard le Bovier de (1740). Éloges des Académiciens; avec l'Histoire de l'Académie royale des sciences en MDCXCIX, La Haye, van der Kloot, 2 vol.

Fournier, Jean-Marie et Raby, Valérie (2006). « Formes et usages du discours historiographique chez les grammairiens français », Histoire Épistémologie Langage 28/1, 51-75.

Puech, Christian (2006). "Pour une histoire de la linguistique dans l'histoire de la linguistique », Histoire Épistémologie Langage 28/1, 9-24.

Savatovsky, Dan (2006). « Meillet historiographe du comparatisme », Histoire Épistémologie Langage 28/1, 89-104.

Schmitter, Peter et van der Wal, Marijke (eds) (1998). Metahistoriography. Theoretical aspecs of the Historiography of linguistics, Münster, Nodus Publikationen.

Tabouret Keller, Andrée (éd.) (1997). Le nom des langues I, les enjeux de la nomination des langues, Louvain-la-Neuve, Peeters.

Zangara, Adriana (2007). Voir l'histoire : théories anciennes du récit historique, Paris, J. Vrin / EHESS 